\section{Distribution of diaphragm blood flow during sevoflurane anaesthesia in dogs}

Tohru Ide MD, Tetsuo Kochi MD,*

Kazuhiko Lijima MD, Tadanobu Mizuguchi MD
Purpose: The purpose of this study was to determine the effect of increasing the concentrations of sevoflurane anaesthesia on the distribution of diaphragm blood flow (Qdi) in ten dogs during mechanical ventilation.

Methods: Animals were divided into two groups, sevoflurane $(n=6)$ and time control $(n=4)$ groups. Blood flow to the crural and the costal diaphragm ( $\dot{Q}_{c r u}, \dot{Q}$ cost) was determined by the hydrogen clearance technique at 0,0.5, 1.0 and 1.5 minimum alveolar concentration (MAC) of sevoflurane after a 30 min period of steady-state conditions. Cardiac output $(C O)$ and the mean arterial blood pressure $(M B P)$ were also measured.

Results: Sevoflurane anaesthesia caused a reduction in $\mathrm{CO}$ $\left(L \cdot \mathrm{min}^{-1}\right)$ from a control value of $1.51 \pm 0.21$ 10 $1.38 \pm 0.1$ (0.5 MAC), $1.09 \pm 0.15$ (1.0 MAC) and $0.98 \pm 0.12$ (1.5 MAC) (Mean \pm SD). Mean blood pressure, $\dot{Q} c r u$ and $\dot{Q}$ cost also decreased with increasing depth of anaesthesia. In addition, the decrease of $\dot{Q} c r u$ was greater than that of $\dot{Q} c o s t$ at all levels of $M B P$ and $C O$. No change occurred in these variables in the time control group.

Conclusion: Sevoflurane anaesthesia changes the distribution of $\dot{Q} d i$ with a greater reduction occurring in $\dot{Q} c r u$ than in Qcost.

\section{Key words}

ANAESTHETICS, VOLATILE: sevoflurane;

BLOOD: flow, diaphragm;

MEASUREMENT TECHNIQUES: blood flow, hydrogen

clearance;

MUSCLE, SKELETAL: diaphragm.

From the Department of Ancsthesiology, School of Medicine, Chiba University, 1-8-1 Inohana, Chuo-ku, Chiba-shi, Chiba, 260 Japan and the *Department of Anesthesiology, National

Cancer Hospital East, Chiba, Japan.

Address correspondence to: Dr. Tohru Ide, Department of Anesthesiology, School of Medicine, Chiba University, 1-8-1

Inohana, Chuo-ku, Chiba-shi, Chiba, 260 Japan.

Phone: +81(43) 222-7171. Fax: +81(43) 226-2156.

Accepted for publication 21 st September, 1995.
Objectif: Déterminer les effets de l'augmentation de la concentration du sévoflurane sur la distribution du débit sanguin diaphragınatique (Qdi) sur dix chiens pendant la ventilation automatique.

Méthodes: Les animaux ont été répartis en deux groupes, sévoflurane $(n=6)$ et contrôle $(n=4)$. Le débit sanguin au diaphragme crural et costal ( $\left.\dot{Q}_{c r u}, \dot{Q} c o s t\right)$ a été déterminé avec la technique de clairance de l'hydrogène aux concentrations alvéolaires minimales (MAC) de 0,05, 1,0 et 1,5 de sévoflurane après 30 min de stabilisation. Le débit cardiaque $(D C)$ et la pression artérielle moyenne (PĀM) ont aussi été mesurès.

Résultats: L'anesthésie au sévoflurane a provoqué une baisse du $D C\left(L \cdot \min ^{-1}\right)$ comparativement à la valeur de contrôle de $1,51 \pm 0,21$ à $1,38 \pm 0,1$ (à $0,5 \mathrm{MAC})$, à $1,09 \pm 0,15$ (à 1,0 $M A C$ ) et à $0,98 \pm 0,12(a ̀$ l,5 MAC) (moyenne $\pm E T)$. La pression artérielle moyenne, le $\dot{Q} c r$ et le $\dot{Q}$ cost ont aussi diminué avec l'approfondissement de l'anesthésie. En outre, la diminution du Q́cru a été plus importante que celle du Q́ cost à tous les niveaux de $P \overline{A M}$ el de DC. On n'a noté aucun de ces changements dans le group contrôle.

Conclusion: L'anesthésie au sévoflurane modifie la distribution du $\dot{Q}$ di mais le $\dot{Q} c r u$ diminue plus que le $\dot{Q}$ cost.

We have recently demonstrated that deep sevoflurane anaesthesia has a moderate depressant effect on diaphragmatic function. Electromyographic recording indicated that the contractile function of the crural part of the diaphragm was depressed more than the costal part.' It has been recently demonstrated that inhalational anaesthetics depresses the function of diaphragm. ${ }^{2-7}$ The exact mechanism underlying this effect remains unclear, although a reduction in energy supply to the diaphragm due to a decrease in blood flow during inhalational anaesthetics may depress diaphragmatic function. Furthermore, the different sensitivity of the two parts of diaphragm to sevoflurane may be due to a maldistribution of blood flow to the diaphragm (Qdi). A change in Qdi caused by inhalational anaesthetics has been reported previously, although such change seems to depend on species, the type and dose of anaesthesia, and the venti- 
latory state. Gelman et al. ${ }^{8}$ reported that isoflurane at 1.0 minimum alveolar concentration (MAC) increased Qdi, whereas 1.0 MAC halothane decreased Qdi in mechanically ventilated dogs. Seyde and Longnecker ${ }^{9}$ demonstrated that both halothane and isoflurane reduced Qdi. In these two studies the radiolabelled microspheres technique were used to measure Q́di. Leon et al. ${ }^{10}$ showed a different response in capillary density between halothane and isoflurane, using direct visualization of the diaphragmatic microvascular network. It has also been reported that the distribution of blood flow to the costal and crural diaphragm can be altered by ventilatory state and body position. ${ }^{11-13}$ However, the effect of inhalational anaesthetics on the distribution of Qdi within the two parts of diaphragm has not been established. The present study was designed to assess the effect of sevoflurane on the distribution of Qdi using the hydrogen clearance technique.

\section{Methods}

Institutional approval for the experiment was obtained from Animal Care and Use Committee of Chiba University School of Medicine. A total of ten mongrel dogs, $10-16 \mathrm{~kg}$, were anaesthetized with pentobarbitone $30 \mathrm{mg} \cdot \mathrm{kg}^{-1}$ iv. Supplemental doses $\left(2 \mathrm{mg} \cdot \mathrm{kg}^{-1} \cdot \mathrm{hr}^{-1}\right)$ were given subsequently to sustain anaesthesia adequate to abolish spontaneous motor activity during the experiment. The femoral artery was cannulated to monitor arterial blood pressure and to withdraw blood samples for measurement of arterial blood gas tensions using the IL blood gas analyzer (model 1302, Instrumentation Laboratories). The femoral vein was also cannulated for the administration of fluids and bicarbonate to correct acidosis, whenever necessary. Rectal temperature was continuously monitored and maintained at $37-38^{\circ} \mathrm{C}$ using a heating lamp.

Animals were randomized into two groups of six and four animals each; the sevoflurane $(n=6)$ and time control $(n=4)$ groups. Surgical preparation of each animal included tracheal intubation with a cuffed endotracheal tube and paralysis with pancuronium bromide 0.02 $\mathrm{mg} \cdot \mathrm{kg}^{-1}$. Following tracheostomy, a tracheal cannula was placed and mechanical ventilation was established with $\mathrm{FIO}_{2}$ of 0.95 monitored with an expired gas monitor (IH2IA, Sanei, Japan). The dogs were placed in the supine position and the frequency and stroke volume of the ventilator adjusted to keep $\mathrm{PaCO}_{2} 30$ and $35 \mathrm{mmHg}$.

Diaphragm blood flow (Qdi) was determined by the hydrogen clearance technique using a UH meter (PHG201, Unique Medical, Japan), theoretically based on Kety's approach to blood-tissue exchange of inert gases. The validity of the method for measuring local blood flow in tissues has been examined and confirmed by
Aukland $e t a l . .^{14}$ and by Neely et al ${ }^{15}$ The basic paradigm of the method is the detection of hydrogen concentration in tissues reflected by the polargraphic technique which is based on the principle that the oxidation reaction, $\mathrm{H}_{2} \rightarrow 2 \mathrm{H}^{+}+2 \mathrm{e}^{-}$, generates electrons. Hydrogen is metabolically inert and not normally present in body tissues. In addition, the pulmonary circulation should rapidly remove it from arterial blood because of its low water:gas partition coefficient of 0.018 . Therefore, hydrogen fulfills the major criteria for tracer clearance studies of blood flow. Hydrogen administered either by respiration or intra-arterially is transported to the surface of the positively polarized platinum electrode where it produces a current proportional to the hydrogen concentration at the electrode. As hydrogen is cleared from the tissue and removed from the blood by the lungs, tissue hydrogen clearance is reflected by a decreasing current that is related to the rate of blood flow at the site of the electrode.

In this study, platinum electrodes (standard wire, Unique Medical, Japan) were implanted through a small laparotomy in the anterior portion of the crural part of the diaphragm near the central tendon, and the anterior portion of the costal part (away from the zone of apposition) in the left hemidiaphragm and then the laparotomy was closed tightly in layers. For measurement of Qdi, the lungs were ventilated with a mixture of $5 \%$ hydrogen, $95 \%$ oxygen for five minutes. Hydrogen washout curves were plotted on a multichannel recorder (R-12, Rikadenki, Japan) from the start of hydrogen inspiration. The half-time was measured from a semi-logarithmic plot of hydrogen concentration against time. To measure smooth curves and a stable baseline, no other measurements were made during the plotting of hydrogen washout curves.

To measure cardiac output (CO), an electromagnetic flow probe (model FB, 15-20 mmID, Nihon Koden, Japan) was placed in the ascending aorta through a sternotomy. This was followed by closure of the chest. The proximal end of the cable was connected to an electromagnetic flowmeter (MFV-3100, Nihon Koden, Japan) with a flow measurement range from $0.2 \mathrm{ml} \cdot \mathrm{min}^{-1}$ to $19.99 \mathrm{~L} \cdot \mathrm{min}^{-1}$.

A 60 -min equilibration period was allowed after completion of surgery. Measurement of $\mathrm{CO}$ and the blood flow to the crural and costal diaphragm (Q́cru, Qcost) were measured during the control (no sevoflurane anaesthesia) and at three levels of anaesthesia in each animal $0.5,1.0$ and $1.5 \mathrm{MAC}$ sevoflurane, following a 30 min period of steady state condition at each concentration. Sevoflurane was administered using a continuous flow vaporizer (Acoma, Japan). The end-tidal concentration of the anacsthetic agent was measured with 
TABLE I Arterial blood pH, $\mathrm{PCO}_{2}\left(\mathrm{PaCO}_{2}\right)$, and $\mathrm{PO}_{2}\left(\mathrm{PaO}_{2}\right)$ in the sevoflurane and the time control groups

\begin{tabular}{llll}
\hline & $p H$ & $\begin{array}{l}\mathrm{PaCO}_{2} \\
(\mathrm{mmHg})\end{array}$ & $\begin{array}{l}\mathrm{PaO}_{2} \\
(\mathrm{mmHg})\end{array}$ \\
\hline Sevoflurane group & & & \\
$0 \quad \mathrm{MAC}$ & $7.416 \pm 0.300$ & $34.6 \pm 1.8$ & $460 \pm 5.8$ \\
$0.5 \mathrm{MAC}$ & $7.415 \pm 0.230$ & $35.4 \pm 2.6$ & $472 \pm 4.2$ \\
$1.0 \mathrm{MAC}$ & $7.407 \pm 0.146$ & $36.4 \pm 1.2$ & $424 \pm 4.8$ \\
$1.5 \mathrm{MAC}$ & $7.399 \pm 0.380$ & $35.2 \pm 1.4$ & $460 \pm 6.2$ \\
& & & \\
$7 \mathrm{ime} \mathrm{control} \mathrm{group}$ & & & \\
$0 \mathrm{hr}$ & $7.400 \pm 0.120$ & $36.8 \pm 3.2$ & $450 \pm 9.2$ \\
$1 \mathrm{hr}$ & $7.412 \pm 0.124$ & $35.8 \pm 2.8$ & $464 \pm 5.9$ \\
$2 \mathrm{hr}$ & $7.406 \pm 0.242$ & $34.8 \pm 4.2$ & $429 \pm 8.8$ \\
$3 \mathrm{hr}$ & $7.389 \pm 0.302$ & $38.9 \pm 4.3$ & $424 \pm 7.8$ \\
\hline
\end{tabular}

Values are mean \pm SD.

$\mathrm{PaCO}_{2}=$ arterial carbon dioxide tension; $\mathrm{PaO}_{2}=$ arterial oxygen tension; $\mathrm{MAC}=$ minimum alveolar concentration.

an anaesthetic gas monitor (Capnomac, Datex). The sequence of changing sevoflurane concentration $(0.5$, 1.0 and $1.5 \mathrm{MAC}$ ) was randomized among animals. In the time control group, to verify the stability of the preparation, measurements were made every $60 \mathrm{~min}$ for four hours after the initial equilibration period, namely the first $(0 \mathrm{hr})$, second ( $1 \mathrm{hr})$, third (2 hr), and fourth measurements $(3 \mathrm{hr})$. Arterial blood gas tensions and mean arterial blood pressure (MBP) were determined at the end of each run. All values were expressed as mean \pm SD. Statistical analysis was performed using analysis of variance and Tukey's test.

\section{Results}

Mean ( $\pm \mathrm{SD}$ ) values of arterial blood $\mathrm{pH}, \mathrm{PO}_{2}\left(\mathrm{PaO}_{2}\right)$, $\mathrm{PCO}_{2}\left(\mathrm{PaCO}_{2}\right)$ under each experimental condition are summarized in Table I. These variables remained unchanged at all levels of anaesthesia. Increasing the depth of sevoflurane anaesthesia caused reduction of CO and decreased MBP, Qcru and Q́cost (Table II). To quantify the relationship between MBP and Qdi (Q́cru and Q́cost), or CO and Qdi at all levels of sevoflurane anaesthesia, values were expressed as percentage of the control. The Figure depicts the CO-Qdi (left panel) and the MBP-Qdi (right panel) relationships. The reduction in Qcru was greater than that of Q cost at any given level of $\mathrm{MBP}$ and $\mathrm{CO}$. In the control group, there were no changes in MBP, CO, Q́cru or Q́cost with time.

\section{Discussion}

The main findings of the present study were that, under conditions of controlled mechanical ventilation without pacing, sevoflurane reduced Qdi in a dose-dependent manner. In addition, the depressive effect of sevoflurane on Qdi was greater in Q́cru than Q́cost at any given circulatory variables of $\mathrm{CO}$ and MBP. The percent changes in CO and MBP correlated well with those of Qdi.

Previous studies of respiratory muscle blood flow have concentrated on differences between various muscle groups. During spontaneous breathing, Qdi is known to be greater than that of the external intercostal muscles, which in turn are better perfused than the internal intercostal muscles. In this experiment, we studied the distribution of $\dot{Q}$ di during sevoflurane anaesthesia under conditions of controlled mechanical ventilation. The Qdi during mechanical ventilation after paralysis was the minimum Qdi under various conditions, and was considered the basic value with changes of Qdi as the work of breathing varied.

Diaphragmatic blood flow has been measured using a flow meter, timed collection of venous blood or radiolabelled microspheres techniques. Nichols et al. ${ }^{16}$ have phrenic arterial blood flow (Q́pa) with a doppler flow probe and Qdi using the radiolabelled microspheres technique and demonstrated a good correlation between changes in Q̀pa and changes in regional and hemispheric Qdi over a wide range of MBP under conditions of supramaximal phrenic nerve stimulation. However, each hemidiaphragm receives its blood supply from the phrenic artery, the internal mammary artery, and the 8th-12th intercostal arteries. Measurement of $\dot{Q} \mathrm{di}$ is complicated by the presence of a local network of arteries and extensive anastomoses between the three arteries on the same side and the opposite hemidiaphragm. For this reason, regional difference in Qdi are not likely to be detected by methods measuring arterial blood flow or timed collection of venous flow, and these techniques may not be suitable to our study in which the distribution of Qdi was studied. Recently, the radiolabelled microspheres technique has been widely used to measure Qdi. Although this technique has many advantages, we could not use this technique in our study because of our facility limitations.

We used the hydrogen clearance technique to measure Qdi. This method has certain advantages over other blood flow monitoring techniques. For example, multiple flow determinations can be obtained using the same tissue site over long periods of time, thus allowing investigation of changes in the distribution of Q di within the hemidiaphragm during sevoflurane anaesthesia. The Qdi can be estimated from the clearance rate of hydrogen, independent of the absolute amplitude of the hydrogen signal. In addition, this is among the least expensive blood flow monitoring techniques. It should be noted, however, that several limitations of the method have been pointed out. First, the method measures the aver- 
TABLE II Mean arterial blood pressure (MBP), cardiac output (CO), and the crural and costal diaphragmatic blood flow (Qdi cru, Qdi cost) in the sevoflurane and the time control groups.

\begin{tabular}{|c|c|c|c|c|}
\hline & $\begin{array}{l}M B P \\
(m m H g)\end{array}$ & $\begin{array}{l}C O \\
\left(L \cdot \min ^{-1}\right)\end{array}$ & $\begin{array}{l}\dot{Q}_{c r u} \\
\left(\mathrm{ml} \cdot 100 \mathrm{~g} \cdot \mathrm{min}^{-1}\right)\end{array}$ & $\begin{array}{l}\dot{Q} \operatorname{cost} \\
\left(\mathrm{ml} \cdot 100 \mathrm{~g} \cdot \mathrm{min}^{-1}\right)\end{array}$ \\
\hline \multicolumn{5}{|c|}{ Sevoflurane group } \\
\hline 0 MAC & $123 \pm 5.0$ & $1.51 \pm 0.21$ & $20.8 \pm 3.4$ & $26.2 \pm 4.0$ \\
\hline $0.5 \mathrm{MAC}$ & $116 \pm 5.4$ & $1.38 \pm 0.11^{*}$ & $18.2 \pm 2.0$ & $24.6 \pm 5.4$ \\
\hline $1.0 \mathrm{MAC}$ & $90 \pm 3.0 \dagger$ & $1.09 \pm 0.15+\$$ & $14.6 \pm 4.2 \dagger \S$ & $21.8 \pm 3.9^{*}$ \\
\hline $1.5 \mathrm{MAC}$ & $74 \pm 2.4 \ddagger \S \mathbb{I}$ & $0.98 \pm 0.12 \S 9$ & $12.8 \pm 3.2 \ddagger \S 4$ & $18.8 \pm 2.8 \dagger \S$ \\
\hline \multicolumn{5}{|c|}{ Time control group } \\
\hline $0 \mathrm{hr}$ & $123 \pm 4.5$ & $1.49 \pm 0.16$ & $20.3 \pm 1.6$ & $26.3 \pm 1.9$ \\
\hline $1 \mathrm{hr}$ & $123 \pm 3.4$ & $1.50 \pm 0.14$ & $21.5 \pm 1.7$ & $26.4 \pm 2.4$ \\
\hline $2 \mathrm{hr}$ & $124 \pm 3.9$ & $1.47 \pm 0.15$ & $21.7 \pm 1.2$ & $26.8 \pm 1.3$ \\
\hline $3 \mathrm{hr}$ & $122 \pm 1.4$ & $1.48 \pm 0.16$ & $22.0 \pm 1.6$ & $27.4 \pm 1.6$ \\
\hline
\end{tabular}

Values are mean \pm SD.

MBP = mean arterial blood pressure: $\mathrm{CO}=$ cardiac output; $\dot{\mathrm{Q}} \mathrm{cru}=$ blood llow to the crural diaphragm; Qcost = blood flow to the costal diaphragm $* P<0.05$ vs Control. $\dagger P<0.05$ vs 0.5 MAC. $\ddagger P<0.05$ vs. $1.0 \mathrm{MAC}$. $\$ P<0.01$ vs Control. $\llbracket P<0.01$ vs 0.5 MAC.
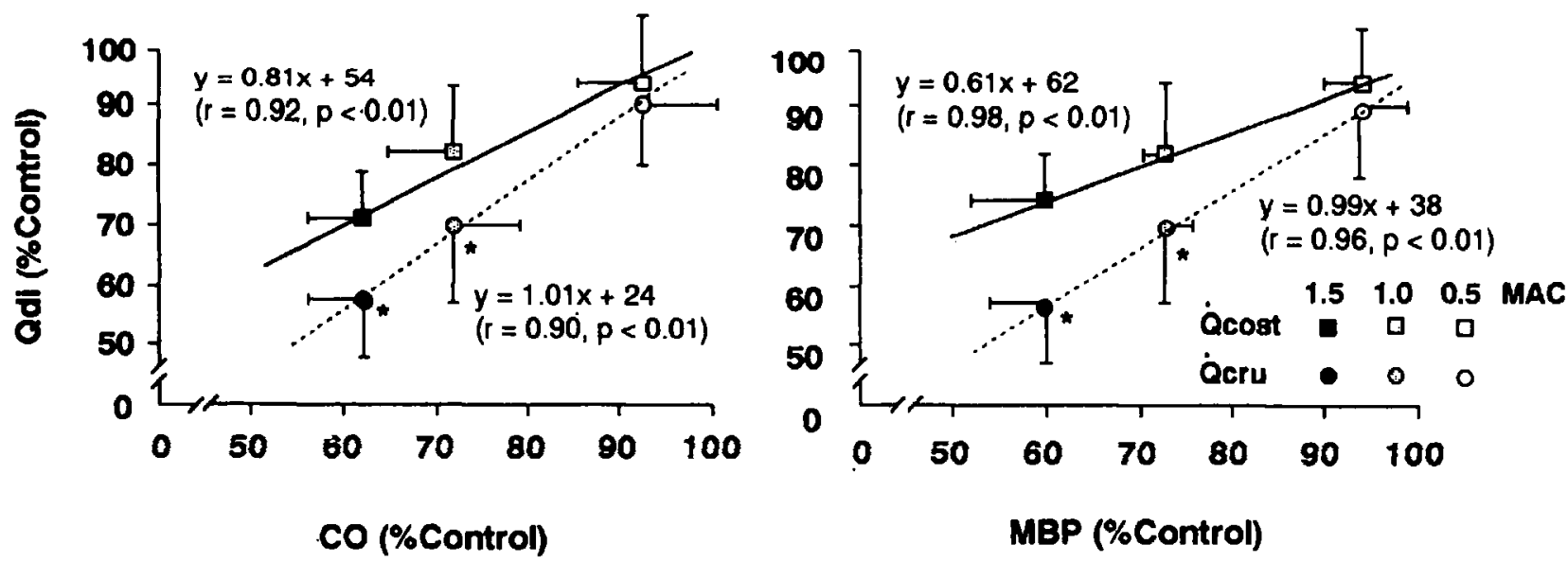

FIGURE Relationship between diaphragmatic blood flow (Qcru, $\mathbf{Q} c o s t)$ and cardiac output (CO) (Left panel), mean arterial blood flow (MBP) (Right panel) during scvoflurane anaesthesia. Values are expressed as the percentage of control value obtained in each animal. Each point represents the mean data with bars indicating 1 SD. $* P<0.05$ vs Q cost.

age flow over several minutes during inspiration and expiration. Second, the hydrogen clearance curves are often polyexponential, raising serious questions concerning the use of a single exponential clearance rate for approximating blood flow. Multi-exponential curves were not recorded in the present study, and the first one to two minutes of the clearance data were discarded to circumvent artifacts due to recirculated arterial hydrogen.

Surgical preparation of the diaphragm for flow mea- surement may damage the local vascular network. However, in the present model, the diaphragm itself was intact except for the implantation of small electrodes within muscle tissue. The diaphragm was not affected by the surgical preparation, which involved the abdominal muscle and sternum. The sternotomy was performed in the upper part of the sternum to exclude the possibility of any lesion to the diaphragmatic vascular branches, although internal mammary arteries might be affected by the preparation. 
Lockhat et al. ${ }^{17}$ have demonstrated, in spontaneously breathing dogs using the radiolabelled microspheres technique, that occlusion of the thoracic aorta reduces Q $c r u$ while not affecting Q $\operatorname{cost}$. These data suggest that the phrenic artery is the major blood supply to the crural part, whereas intercostal and internal mammary arteries can maintain flow to the costal part of the diaphragm. Our data suggest that intercostal and internal mammary blood flow may be preserved during sevoflurane anaesthesia compared with phrenic artery blood flow since there was a trend towards a greater reduction in Qcru than Qcost. Several possible mechanisms may explain the different response of $\dot{Q}$ cru and $\dot{Q} c o s t$ to increasing depth of sevoflurane anaesthesia. Brancatisano et al. ${ }^{13}$ suggested that the level of anaesthesia affected the blood flow during different respiratory modes, e.g., paralyzed state vs spontaneous breathing. In the present experiment, the lungs were mechanically ventilated, therefore the breathing pattern and work load did not affect Qdi. Differences in energy requirement for contraction between the crural and costal diaphragm have also been described." These differences may enhance a state of maldistribution of Qdi during sevoflurane anaesthesia. The mammalian diaphragm is composed of three types of muscle fibres, slow-twitch oxidative, fasttwitch oxidative, and fast-twitch glycolytic muscle fibres. In rat muscles, the specific blood flow to slowand fast-twitch red muscles differs because of the different oxidative capacity which is higher in fast-twitch than slow twitch fibres. Therefore, marked regional variation of fibre type distribution within the diaphragm may be associated with corresponding variation in the distribution of blood flow during sevoflurane anaesthesia. As Brancatisano et al. pointed out, ${ }^{13}$ it is possible that the weight of the abdominal contents acting on the dependent dorsal parts could narrow blood vessels, particularly the phrenic artery which is exposed in the supine animal, thus displacing or "squeezing" blood toward the ventral regions in a dose dependent manner.

Hussein et al..$^{16}$ have demonstrated that $\dot{Q}$ di is well autoregulated between MBP of 70 and $120 \mathrm{mmHg}$ during quiet breathing. Our data demonstrated that Qdi was directly related to MBP or $\mathrm{CO}$, suggesting that sevoflurane anaesthesia may impair the autoregulatory mechanisms.

Since the dogs were anaesthetized with pentobarbitone in the present experiment, we examined the combined effects of sevoflurane and pentobarbitone. Although no data are available regarding the effect of pentobarbitone on Qdi, our control study did not demonstrate any effect of pentobarbitone on MBP, CO, or Qdi (Q'cru and Qcost), suggesting that the changes in these variables observed during sevoflurane exposure were mainly related to sevoflurane or represented the effects of deeper anaesthesia.

Several investigators have measured Qdi with the Kety-Schmidt technique using inert tracer gas or radiolabelled microspheres technique and demonstrated a linear relationship between electromyographic activity of the diaphragm and Qdi when the work of breathing is increased with inspiratory resistive loading of hypercapnia. ${ }^{17-19}$ Therefore our previous results demonstrating a preferential depressive effect by sevoflurane on the electromyographic activity of crural diaphragm compared with the costal activity' may, in part, be due to the effect of sevoflurane on the distribution of Qdi.

In summary, this study demonstrates that in the paralyzed dog during mechanical ventilation, Qdi is distributed inhomogeneously within the diaphragm. During sevoflurane anaesthesia, a greater reduction occurred in Q'cru than in Q $c o s t$.

\section{References}

1 Ide $T$, Kochi T, Isono S, Mizuguchi T. Effect of sevoflurane on diaphragmatic contractility in dogs. Anesth Analg 1992; 74: 739-46.

2 Clergue F, Viires $N$, Lemesle $P$, Aubier $M$, Viars $P$, Pariente $R$. Effects of halothane on diaphragmatic muscle function in pentobarbital-anesthetized dogs. Anesthesiology 1986; 64: 181-7.

3 Dureuil R, Viirès $N$, Nivoche $Y$, et al. Different effects of halothane on diaphragm and hindlimb muscle in rats. $\mathrm{J}$ Appl Physiol 1987; 63: 1757-62.

4 Veber B, Dureuil B, Viires N, Aubier M, Pariente R, Desmonts $J M$. Effects of isoflurane on contractile properties of diaphragm. Anesthesiology 1989; 70: 684-8.

5 Kochi T, Ide T, Isono S, Mizuguchi T, Nishino T. Different effects of halothane and enflurane on diaphragmatic contractility in vivo. Anesth Analg 1990; 70: 362-8.

6 Ide T, Kochi T. Isono S, Mizuguchi T. Diaphragmatic function during sevoflurane anaesthesia in dogs. Can J Anaesth 1991; 38: 116-20.

7 Ide T, Kochi T, Isono S, Mizuguchi T. Diaphragmatic activity during isoflurane anaesthesia in dogs. Acta Anaesthesiol Scand 1993; 37: 253-7.

8 Gelman S, Fowler KC, Smith LR. Regional blood flow during isoflurane and halothane anesthesia. Anesth Analg 1984; 63: 557-65.

9 Seyde WC, Longnecker $D E$. Anesthetic influences on regional hemodynamics in normal and hemorrhaged rats. Anesthesiology 1984; 61: 686-98.

10 Leon A, Boczkowski J, Dureuil B, Vicaut E, Aubier M, Desmonts J-M. Diaphragmatic microcirculation during halothane and isoflurane exposure in pentobarbital-anesthetized rats. J Appl Physiol 1992; 73: 1614-8.

11 Soust $M$, Walker AM, Wilson FE, Berger PJ. Origins and 
regional distribution of blood flow to the respiratory muscles in conscious sheep. Respir Physiol 1987; 67: 283-94.

12 Manohar $M$. Costal vs crural diaphragmatic blood flow during submaximal and near-maximal exercise in ponies. J Appl Physiol 1988; 65: 1514-9.

13 Brancatisano A, Amis TC, Tully A, Kelly WT, Engel LA. Regional distribution of blood flow within the diaphragm. J Appl Physiol 1991; 71: 583-9.

14 Aukland K, Bower BF, Berliner RW. Measurement of local blood flow with hydrogen gas. Circ Res 1964; 14: 164-187.

15 Neely WA, Turner MD, Hardy JD, Godfrey WD. Use of the hydrogen electrode to measure tissue blood flow. J Surg Res 1965; 5: 363-9.

16 Nichols DG, Scharf SM, Traystman RJ, Rowbottam JL. Correlation of left phrenic arterial llow with regional diaphragmatic blood flow. J Appl Physiol 1988; 64: 2230-5.

17 Lockhat D, Magder $S$, Roussos C. Collateral sources of costal and crural diaphragmatic blood flow. J Appl Physiol 1985; 59: 1164-70.

18 Hussain SNA, Roussos C, Magder S. Autoregulation of diaphragmatic blood flow in dogs. J Appl Physiol 1988; 64: 329-36.

19 Rochester DF, Pradel-Guena M. Measurement of diaphragmatic blood flow in dogs from xenon 133 clearance. J Appl Physiol 1973; 34: 68-74. .

20 Rochester $D F$, Betrini $G$. Diaphragmatic blood flow and energy expenditure in the dog: effects of inspiratory airflow resistance and hypercapnia. J Clin Invest 1976; 57 : $66 I-72$.

21 Soust MJ, Walker AM, Berger PJ. Blood flow to the respiratory muscles during hypercapnic hyperpnoea in the newborn lamb. Respir Physiol 1989; 76: 93-106. 